\title{
IJIMI
}

International Journal of Machine Intelligence

ISSN: 0975-2927 \& E-ISSN: 0975-9166, Volume 3, Issue 2, 2011, pp-45-51

Available online at http://www.bioinfo.in/contents.php?id=31

\section{CASHEW KERNELS CLASSIFICATION USING TEXTURE FEATURES}

\author{
NARENDRA V.G. AND HAREESH K.S. \\ Department of CSE, Manipal Institute Of Technology, Manipal, Karnataka, India-576 104 \\ *Corresponding author. Email: narendra.vg@manipal.edu
}

Received: August 10, 2011; Accepted: September 06, 2011

\begin{abstract}
Cashew is a commercial commodity that plays a major role in earning foreign currency among export commodities of India. The sub-sector is getting governmental and non-governmental attention due its significance in commercial activities. The brand patent creation of each cashew varies based on cashew kernels is an issue in current periods.

The purpose of this research work is to explore image processing techniques and approaches on Indian cashew variety identification based on their kernels. Colour is an important quality factor for grading, marketing, and end use of Cashew. Our objective is to develop a cost-effective way to identify the cashew kernels. Such a system would not only facilitate cashew grading but also serve as a quality control tool for processing facilities such as grading and sorting in export industries like cashew.

This paper presents a methodology for identification and classification of cashew kernels white wholes. The texture features are extracted using gray level co-occurrence matrix method. The multilayer feed forward neural network is developed to classify cashew kernels white wholes. An analysis of the efficiency of methodology is found $90 \%$.
\end{abstract}

Keywords: Cashew kernel, Texture Features, Neural Network.

\section{INTRODUCTION}

Cashew (Anacardium occidentale L.) a native of Eastern Brazil was introduced to India by the Portuguese nearly five centuries back. Cashew is the third largest edible tree nut in the world. India has been the dominant player in the cashew trade and has also pioneered the cashew processing and exports. In addition, India is the largest producer of cashew kernels and the largest area holder of this crop in the world.

Cashew processing India has been a pioneer in cashew processing and has been the first country to hit the world market with cashew kernels. The raw cashew nuts collected from the growing areas are moved to the factories for processing. It is ensured that the raw nuts are thoroughly cleaned and are free from all foreign matter before they are processed.

Grades Based on the Size, Shape, and Colour of the cashew kernels are graded into white or scorched wholes, pieces, splits, butts etc. The Government of India Act prescribes 33 different grades of cashew kernels of which only 26 grades are commercially available and exported. W- 320 is the most popular among cashew kernels and also the most available, worldwide. Scorched wholes are another grade of cashew kernels. They are prepared by subjecting the kernels to a longer duration of roasting, whereby they attain a slight brown colour. They however have all the other characteristics of white kernels including nutritional qualities. Butts, splits and pieces are priced low and are ideal for cooking, preparation of sweets and savory snacks and so on.

Today, various kinds of cashews are available in the market with different qualities. To ascertain the quality, grade standard have been designed by considering the colour and the size (weight) of the cashew kernel as important characteristic as shown in Table 1.

Grading of cashew kernels is based on inspection of physical quality attributes such as colour, shape, and size. By using these physical attributes, a trained person determines the cashew kernel of which class (i.e., white wholes). The Table 1 illustrates the Grade designations and definitions of quality of cashew kernels (i.e., white wholes). Reference colour slides are available to assist with the assessment of cashew kernel colour. Despite the training of the grading personnel and the availability of reference slides, the current method for cashew kernel quality evaluation is time consuming, tedious, and inherently inconsistent. An objective and cost-effective computer vision system is needed to segregate cashew kernels. Such a system would not only facilitate cashew grading but also serve as a quality control tool for 
processing facilities such as elevators, seed cleaning plants, and oil mills[1][2].

Computer vision system has proven successful for the objective, online measurement of several agricultural products[2]. Computer vision based cashew grading system is an alternative to the manual, mechanical and optical methods. This method offers automated, high speed, non-destructive and cost effective technique for classification.

Colour and texture features are used to develop a neural network model for classification of different food objects like Idli, Wada, Bonda etc., is discussed in [3][4] a method for classification and gradation of different grains such as Ground nut, Bengal Gram, wheat etc. Geometrical and colour features such as size, shape, RGB etc., are extracted. A neural network model is developed for classification. [3][4](Anami B.S et al, 2009) have discussed a knowledge based nearest mean classifier for classification of Bulk Food objects. L ${ }^{*} a^{*} b^{*}$ colour features are used for classification. [5](R.M. Harlick et al, 1973) have described some easily computable textural features based on gray tone spatial dependencies. They have illustrated the application in category identification tasks of three different kinds of image data. [6](Giaime Ginesu et al, 2004) have worked on detection of foreign bodies in food by thermal image processing. [7](D. Patel et al, 1994) have described a monitoring system, which detects contaminants such as pieces of stone or fragments of glass in foodstuffs. [8](M Barni et.al, 1995) have described vision based intelligent perception system for making automated inspection of chicken meat feasible. The defects are classified by comparing their features against defect description container in a reference database. [9](Bin Zhu et al, 2007) have developed an automated inspection of apple quality based on geometric or statistical features. Paper introduces a Gabor feature based kernel principal component analysis (PCA) method by combining Gabor wavelet representation of apple images and the kernel PCA method for apple quality inspection using nearinfrared (NIR) imaging.

[10](Dong-chen he and Li Wang, 1990) have described aset of textural measures derived from the texture spectrum. The proposed features are used to extract textural information of an image using gray-level cooccurrence matrix.

[11](Cheng-Jin Du and Da-Wen Sun 2005) have developed an automated classification system of pizza sauce spread using colour vision and support vector machines (SVM).

The image transformed from red, green, and blue (RGB) colour space to hue, saturation, and value (HSV) colour space. A vector quantifier is designed to quantify the HS (hue and saturation) space to 256-dimension, and the quantified colour features of pizza sauce spread were represented by colour histogram. [12](Zhao-yan Liu et.al 2005) have described image analysis algorithm based on colour and morphological features for identifying different varieties of rice seeds. Seven colour features and fourteen morphological features are used for discriminate analysis. A two-layer neural network model is developed for classification.

Most of the researchers have focused on classification of fruits, meat, processed food like pizza etc., from their digital images. The present work pertains to classification of cashew kernel white whole based on textural features.

\section{MATERIALS AND METHODS}

\section{Image Acquisition}

The samples of whole cashews of different grades, used in this study were collected from Balaji Exports Jarkal Karkal Tq. one of the cashew production companies of Karnataka. Images for Cashew kernels are acquired using a digital camera (Sony 7.2 Mega Pixels with optical zoom 4x) in a room with a proper control of lighting intensity. Distances and heights between camera and cashew kernel/cashew kernels were fixed for all the samples. A block paper was used as background surface to facilitate and simplify the further task. The images of each cashew kernel sample are captured in different angles. i.e., top, front, left, and right views are collected. The sample digital images of different samples of cashew kernels are shown in "Fig.(1)".

The texture features such as Contrast, Correlation, Entropy, Energy, Homogeneity, Dissimilarity, Smoothness, Cluster Shade, Cluster Performance, Angular Second Moment, Third Moment, Mean, Variance, Standard Deviation, and Maximum Probability are extracted using gray level co-occurrence matrix. The extracted features are used to train the developed neural network model. The developed neural network model is tested for recognition and classification of different cashew kernel white whole samples[3][4]. The block diagram representing different phases of the work is given in "Fig.(2)".

\section{TEXTURE FEATURES EXTRACTION}

Texture is one of the most important defining characteristics of an image. It is characterized by the spatial distribution of gray levels in a neighborhood. There are different types of texture feature extraction methods like statistical, geometrical, and model based and signal processing reported in the literature. Statistical methods analyze the spatial distribution of gray values by computing local features at each point in the image and deriving a set of statistics from the distributions of the local features. Geometrical methods try to describe the primitives and the rules governing their spatial organization by considering texture to be composed of texture primitives. The structure and organization of the primitives can be presented using Voronoi tessellations. Model based texture analysis methods are based on the construction of an image model that can be used not only to describe texture, but also to synthesize it. The model parameters capture the essential perceived qualities of texture. Signal processing methods 
analyze the frequency content of the image. For food processing, the most widely used approaches are statistical including pixel-value run length method and the co-occurrence matrix method[3].

\section{Gray level Co-occurrence Matrix (GLCM) Method}

GLCM is a two dimensional matrix of frequencies at which two pixels, separated by a certain vector, occurs in the image. i.e., the GLCM is a tabulation of how often different combination of pixel brightness values (gray levels) occur in an image. The distribution in the matrix depends on the angular and distance relationship between pixels. Varying the vector used allows the capturing of different texture characteristics. Once the GLCM has been created, various features can be computed from it. After creating GLCM, it is required to normalize the matrix before texture features are calculated. The measures require that each GLCM cell contain a count, but rather a probability[3].

To accomplish texture analysis task, the first step is to extract texture features that most completely embody information about the spatial distribution of intensity variations in the textured image. Texture features derived from GLCM using the formulae given in Table 2[3][4].

Contrast returns a measure of intensity contrast between a pixel and its neighborhood. Contrast is 0 for a constant image. Energy means uniformity, or angular second moment (ASM). The more homogeneous of image, larger the value. When energy equals to 1 , the image is believed to be a constant image. Entropy is a measure of randomness of intensity image. Image with more number of occurrences of particular colour configurations has resulted in higher value of entropy. Local Homogeneity measures the similarity of pixels. Diagonal gray level co-occurrence matrix gives homogeneity of 1.Cluster Shade and cluster prominence are measures of skewness of the matrix, in other words, the lack of symmetry. When cluster shade (CS) and cluster prominence (CP) are high, the image is not symmetry. Maximum Probability gives the maximum occurrence of gray levels which satisfies the relation given in an entropy equation. The steps involved in texture feature extraction are given in Algorithm 1. The algorithm is rotational and scaling invariant. However, the noise free images are used through suitable processing. Hence, it is noise invariant[3].

\footnotetext{
Algorithm 1: Texture features extraction GLCM Method[3].

Input: RGB components of original Image.

Output:15 Texture Features

Start:

Step1: For all the sampled RGB components Derive the Gray Level Co-occurrence Matrices (GLCM)

$P \varphi, d(x, y)$ for four directions $\varphi\left(0^{0}, 45^{\circ}, 90^{\circ}\right.$ and $\left.135^{\circ}\right)$ and $\mathrm{d}=1$ which are dependent on direction $\varphi$

Step2: Compute the co-occurrence matrix, which is independent of direction using equation (1).
}

$$
\mathrm{C}=\overline{1} / 4\left(\mathrm{P}_{0}{ }^{0}+\mathrm{P}_{45}{ }^{0}+\mathrm{P}_{90}{ }^{0}+\mathrm{P}_{135^{0}}\right)_{--(1)}^{-}
$$

Step3: GLCM features namely, mean, contrast etc., are calculated using equations given in Table 2.

Stop

\section{NEURAL NETWORK MODEL}

The multilayer feed forward neural network model with back propagation algorithm for training is employed for classification task as shown in Figure 3. The number of neurons in the input layer is equal to the number of input features (15). The number of neurons in the output layer is equal to the number of categories of cashew kernel(single) white wholes(6).And in hidden layer the number of neurons are 10.

\section{Training and Testing}

The developed neural network is trained with six different cashew kernel white wholes categories namely W-180,W210,W-240,W-320,W-450 and W-500 with 600 samples representing 100 examples of each of six types of cashew kernel white wholes. The texture features of an image are used as the inputs.

In the testing phase, the bulk cashew kernels from untrained set of samples are used to test the trained neural network model for classification. The developed neural network is trained with termination error (TE) 0.01 in 42 epochs the value of learning constant (learning rate LR) used is 0.1 .

\section{Results and discussions}

This section gives results of experiments carried on the developed neural network model. The algorithm was developed for texture feature extraction using GLCM method performed well in the task of extracting texture features from images of different cashew kernels white wholes. The results obtained in this work indicate that the ANNs can in general classify cashew kernels white wholes with success rate of $80 \%$ to $90 \%$. Initial model is developed using only six texture features such as Contrast, Correlation, Energy, Entropy, Homogeneity, and Dissimilarity. The neural network model performance found to be low $(74 \%)$. Since these features are not sufficient for better classification of cashew kernels white wholes, some more features are extracted. Hence fifteen features shown in Table 2 are considered for improving the performance. The accuracies (i.e.90\%) obtained for classification of cashew kernels white wholes when developed neural network is trained with different learning constants and different termination errors. The results obtained in this work indicate that the ANNs can in general classify cashew kernel(single) white wholes. 


\section{Classifier performance}

A test sample set, which was not part of either the training or validation samples, was used to test the neural network classifier trained. The test set included 100 randomly selected cashew kernels. The test results of classification are summarized in Table 3. Almost all $(90 \%)$ of the cashew kernels (single) were correctly classified.

We seen that, using the current features the first 4 grades can be discriminated with no errors. However work still has to be carried out to discriminate between grades ' 450 ' and '500'.

\section{CONCLUSIONS AND FUTURE WORK}

The work gives an efficient model for cashew kernel white wholes identification and classification. The gray level cooccurrence method is found to be an efficient method for texture future extraction. The developed neural network model classifies six different categories of a cashew kernel white wholes with overall $90 \%$ accuracy. In this work gray scale images are considered. The classification accuracy was acquired under laboratory setting, so it had some limits. In future work, a large quantity of colour features of cashew kernels will be investigated for better classification.

\section{REFERENCES}

[1] Balasubramanian D. (2001) Journal of agricultural Engineering Research, 78(3), pp. 291-297.

[2] Brosnan T. and Sun D.W. (2004) Journal of Food Engineering, 61, pp. 3-16.
[3] Anami B.S. and Vishwanath C.Burkpalli (2009) ICGSTGVIP Journal, ISSN: 1687-398X, Volume 9, Issue 4, pp 9-14.

[4] Anami B.S. and Savakar D.G. (2009) International Journal of Artificial Intelligence and Machine Learning Vol 9(1), pp 1-9.

[5] Haralick R.M., Shanmugan K. and Dinestein I. (1973) IEEE Transactions on Systems, Man, Cybernetics, Vol(3), pp. 610-621.

[6] Giaime Ginesu, Daniele D. Giusto, Volker Margner, Peter Meinlschmidt (2004) IEEE Transactions on Industrial Electronics, Vol 51, No 2.

[7] Patel D., Hannah I. and Davies E.R. (1994) Proc of the 12th IAPR international conference E., Volume $1 \mathrm{pp}$ 586-588.

[8] Barni M., Mussa A.W., Mecocci A., Cappelline V., Durrani T.S. (1995) IEEE International Conference on Image Processing Vol 1, pp 450-453.

[9] Bin Zhu, Lu Jiang, Yaguang Luo and Yang Tao (2007) Journal of Food Engineering. Vol 81(4), pp741-749.

[10] Dong-Chen HE and LI Wang (1990) IEEE Trans. Geosciences and remote sensing, vol 28, no 4.

[11]Cheng-Jin Du, Da-wen (2005) Journal Food Engineering. Vol.66(2) pp 137-145

[12] Zhao-yan Liu, Fang Cheng, Yi-bin Ying, \& Xiu-qin Rao (2005) Journal of Zhejiang University, Science B, Vol. 6(11), pp 1095-1100. 
Table -1: Cashew Kernels(White Wholes)

\begin{tabular}{|c|c|l|}
\hline Grade & Count per 454 gms & \multicolumn{1}{|c|}{ General Characteristic } \\
\hline W-180 & $170-180$ & Cashew kernels shall have been obtained through shelling \\
W-210 & $200-210$ & and peeling cashew nuts. Shall have the characteristic \\
W-240 & $220-240$ & shape; shall be white, pale ivory or light ash in colour \\
W-320 & $300-320$ & reasonably dry and free from insect damage, damaged \\
W-450 & $400-450$ & kernels and black or brown spots. The kernels shall be \\
W-500 & $450-500$ & completely free from testa. \\
\hline
\end{tabular}

Table -2 Texture Features Used In The Work

\begin{tabular}{|c|c|}
\hline Property & Formula \\
\hline Contrast & $\sum_{I, J=0}^{n-1} P_{I, J}|i-j|^{2}$ \\
\hline Correlation & $\sum_{i, j=0}^{N-1}\left[\frac{\left(i-\mu_{i}\right)\left(j-\mu_{j}\right)}{\sqrt{\left(\sigma^{2}\right)\left(\sigma^{2}\right)}}\right]$ \\
\hline $\begin{array}{l}\text { Angular } \\
\text { Second } \\
\text { Moment }\end{array}$ & $\sum_{i, j=0}^{N-1} P_{i, j}^{2}$ \\
\hline Energy & $\sqrt{A S M}$ \\
\hline Dissimilarity & $\sum_{i, j=0}^{N-1} P_{i, j}|i-j|$ \\
\hline Entropy & $\sum_{i, j=0}^{N-1} P_{i, j}\left(-\ln P_{i, j}\right)$ \\
\hline Homogeneity & $\sum_{i, j=0}^{N-1} \frac{P_{i, j}}{1+(i-j)^{2}}$ \\
\hline Cluster Shade & $\sum_{i, j=0}^{N-1}\left(\left(i-\mu_{i}\right)+\left(j-\mu_{j}\right)\right)^{3} P_{i, j}$ \\
\hline $\begin{array}{l}\text { Cluster } \\
\text { Performance }\end{array}$ & $\sum_{i, j=0}^{N-1}\left(\left(i-\mu_{i}\right)+\left(j-\mu_{j}\right)\right)^{4} P_{i, j}$ \\
\hline Mean & $\begin{array}{l}\mu_{i} \sum_{i, j=0}^{N-1} i\left(P_{i, j}\right) \\
\mu_{j} \sum_{i, j=0}^{N-1} j\left(P_{i, j}\right)\end{array}$ \\
\hline Variance & $\sigma^{2}=\sum_{i, j=0}^{N-1}\left(P_{i, j}\right)(i-\mu)^{2}$ \\
\hline $\begin{array}{l}\text { Standard } \\
\text { Deviation }\end{array}$ & $\sigma=\sqrt{\sigma^{2}}$ \\
\hline Smoothness & $1-\frac{1}{\left(1+\sigma^{2}\right)}$ \\
\hline $\begin{array}{l}\text { Third } \\
\text { Movement }\end{array}$ & $\sum_{i, j=0}^{N-1}\left(P_{i, j}\right)\left(i-\mu_{i}\right)^{3}$ \\
\hline $\begin{array}{l}\text { Maximum } \\
\text { Probability }\end{array}$ & $\max _{i, j}\left(P_{i, j}\right)$ \\
\hline
\end{tabular}


Table -3: Classification Results for 'White Wholes'

\begin{tabular}{|c|c|c|c|}
\hline $\begin{array}{l}\text { SI. } \\
\text { N0. }\end{array}$ & $\begin{array}{l}\text { Cashew- } \\
\text { Kernel }\end{array}$ & $\begin{array}{l}\text { Classified } \\
\text { Grade }\end{array}$ & $\begin{array}{l}\text { Correct } \\
\text { Classification? }\end{array}$ \\
\hline 1 & 180 & 180 & $\checkmark$ \\
\hline 2 & 180 & 180 & $\checkmark$ \\
\hline 3 & 180 & 180 & $\checkmark$ \\
\hline 4 & 210 & 210 & $\checkmark$ \\
\hline 5 & 210 & 210 & $\checkmark$ \\
\hline 6 & 210 & 210 & $\checkmark$ \\
\hline 7 & 240 & 240 & $\checkmark$ \\
\hline 8 & 240 & 240 & $\checkmark$ \\
\hline 9 & 240 & 240 & $\checkmark$ \\
\hline 10 & 320 & 320 & $\checkmark$ \\
\hline 11 & 320 & 320 & $\checkmark$ \\
\hline 12 & 320 & 320 & $\checkmark$ \\
\hline 13 & 320 & 320 & $\checkmark$ \\
\hline 14 & 450 & 450 & $\checkmark$ \\
\hline 15 & 450 & 450 & $\checkmark$ \\
\hline 16 & 450 & 450 & $\checkmark$ \\
\hline 17 & 450 & 450 & $\checkmark$ \\
\hline 18 & 450 & 450 & $\checkmark$ \\
\hline 19 & 500 & 450 & $X$ \\
\hline 20 & 500 & 450 & $X$ \\
\hline 21 & 500 & 500 & $\checkmark$ \\
\hline 22 & 500 & 450 & $X$ \\
\hline
\end{tabular}




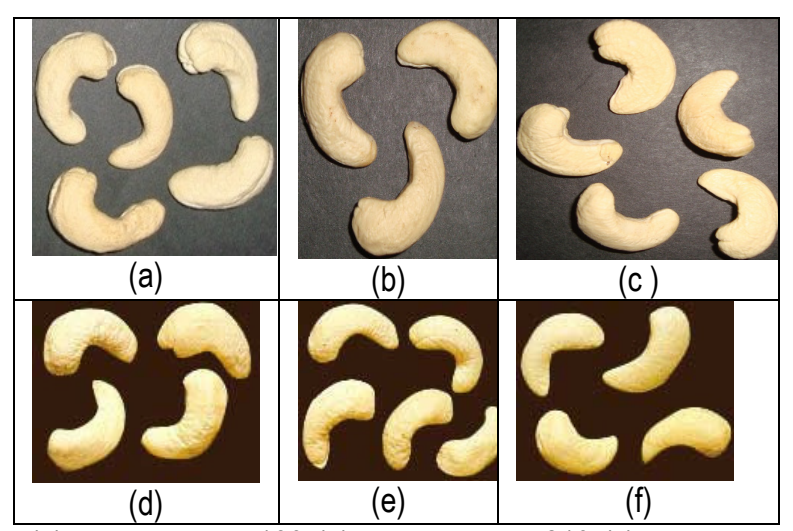

Fig. 1-Images of cashew kernels (a) White wholes-180 (b) White wholes-210 (c) White wholes-240 (d) White wholes-320 (e) White wholes-450 (f) White wholes-500

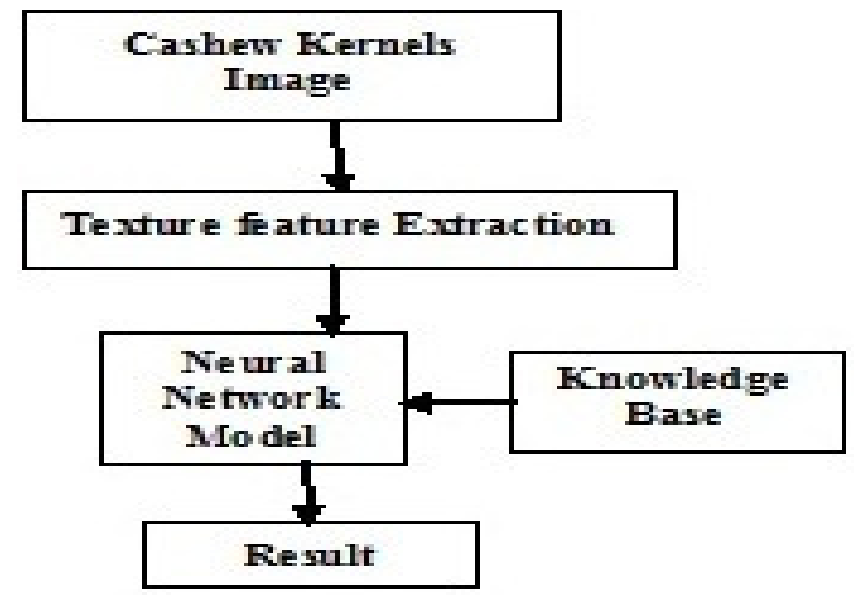

Fig. 2- Block Diagram of the Proposed Methodology[3]

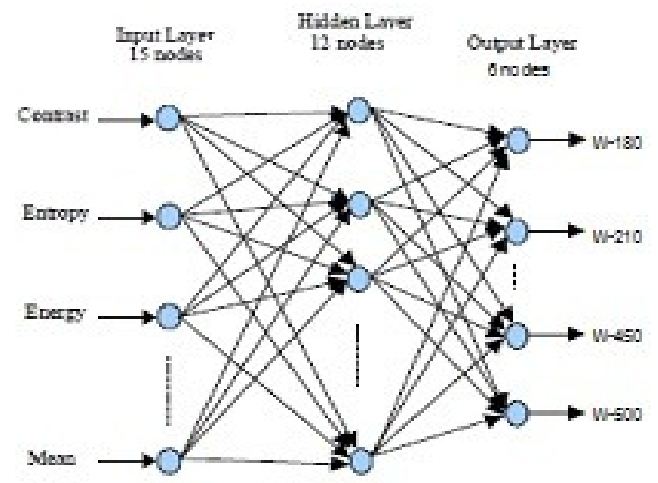

Fig. 3 Neural Nebwod Architecture 